\title{
Treatment of Temporomandibular Joint Disorder by Alloplastic Total Temporomandibular Joint Replacement
}

\author{
Young-Chea Roh, Sung-Tak Lee, Dong-Ho Geum, In-Kyo Chung, Sang-Hun Shin \\ Department of Oral and Maxillofacial Surgery, School of Dentistry, Pusan National University
}

\begin{abstract}
The literature on alloplastic total temporomandibular joint (TMJ) replacement is encouraging, with acceptable improvement of treatment outcomes in terms of both pain level and jaw function. This is a case report on patients who suffered from degenerative joint disease and ankylosis after mandibular condyle fracture or prior TMJ surgery and were treated by TMJ replacement with condyle prosthesis. We obtained good results from the procedures, including total TMJ replacement.
\end{abstract}

Key words: Arthroplasty, Temporomandibular joint, Total disc replacment

\section{Introduction}

Diagnosis and treatment of temporomandibular joint disorder (TMD) is difficult due to functional and anatomical complexity of adjacent structures. In addition, because the temporomandibular joint (TMJ) is involved in pronunciation, mastication, and swallowing, inappropriate treatment may cause complications, such as pain, limitation of mouth opening, myospasm, mandibular displacement, malocclusion, pathologic change, osteonecrosis, facial asymmetry, and ankylosis, which may have a direct impact on the patient's life[1-7]. Especially in post-traumatic TMD, Long and Goss[4] reported that vertical condylar fractures in the joint space may induce pathological changes, such as osteoarthritis, and resultant ankylosis can cause complications such as limited mouth opening. Sanders et al.[5], and Bear and Tankersley[6] also reported that development of TMJ osteoarthritis or ankylosis occurs in most patients with condylar fractures.

Osteoarthritis can be treated not only with drug therapy using nonsteroidal antiinflammatory drugs but also with non-invasive treatments such as behavioral therapy, occlusal therapy, occlusal device, and arthrocentesis. Invasive treatments, such as arthroscopic surgery, discoplasty, discectomy, arthroplasty, and total TMJ replacement, are used when a good therapeutic result cannot not be achieved using such non-invasive treatments, leading to repeated pain, continuous and not improved TMJ dysfunction, or severe anatomical change of TMJ on magnetic resonance imaging and arthrogram[7]. Ankylosis can be treated surgically by condylectomy, gap arthroplasty, and interpositional arthroplasty[8]; however, according to Mercuri[9],

RECEIVED July 30, 2013, REVISED August 27, 2013, ACCEPTED November 26, 2013

Correspondence to Sang-Hun Shin

Department of Oral and Maxillofacial Surgery, School of Dentistry, Pusan National University

20, Geumo-ro, Mulgeum-eup, Yangsan 626-787, Korea

Tel: 82-55-360-5100, Fax: 82-55-360-5104, E-mail: ssh8080@pusan.ac.kr

(C) This is an open access article distributed under the terms of the Creative Commons Attribution Non-Commercial License (http://creativecommons. org/licenses/ by-nc/3.0) which permits unrestricted non-commercial use, distribution, and reproduction in any medium, provided the original work is properly cited. 
these procedures do not provide satisfactory results. Since the introduction of TMJ reconstruction with prosthesis, several studies have reported that improved results could be obtained in terms of both reduced pain and enhanced masticatory function[10].

We report here our experience of satisfactory results using total TMJ replacement with prosthetic condyle and glenoid in patients with pain and limited functioning due to complications, such as degenerative arthritis and ankylosis, after a previous mandibular condyle fracture or prior TMJ surgery for systemic osteoarthritis.

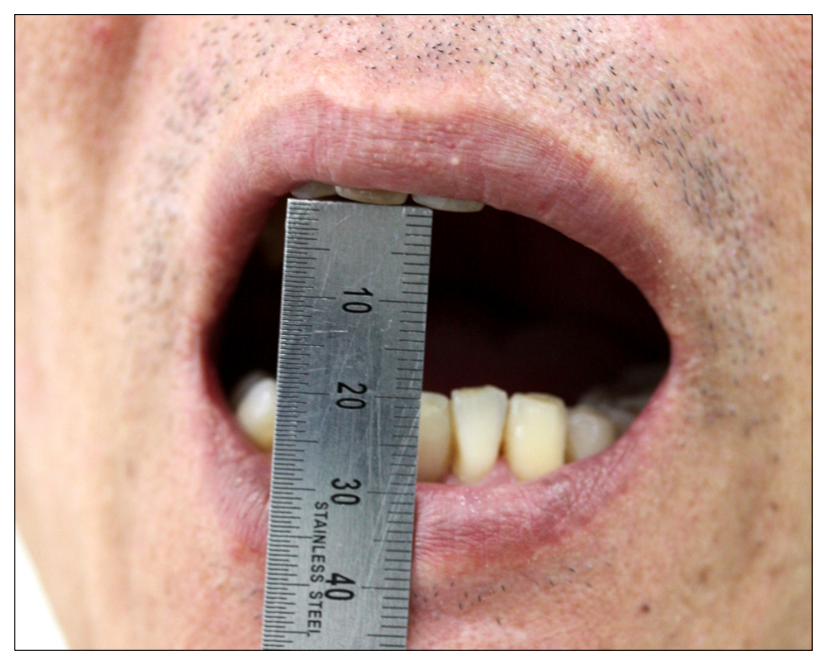

Fig. 1. Preoperative clinical photograph. Maximum interincisal opening was $19 \mathrm{~mm}$.

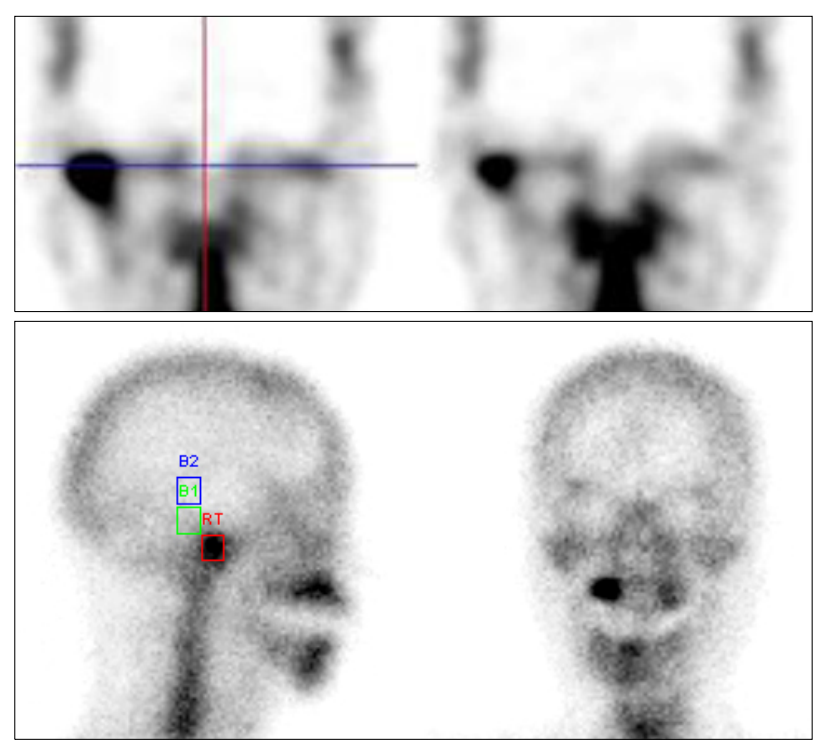

Fig. 3. Bone scan image. Active joint lesion on the right condyle was detected.

\section{Case Report}

\section{Case 1}

A 48-year-old male patient was referred to our department for pain in the anterior auricular region and limited mouth opening. Fourteen months ago, he sustained a bilateral mandibular condylar fracture resulting from a collision. Despite having received physiotherapy at that time, complications had recently recurred. Clinical exami-
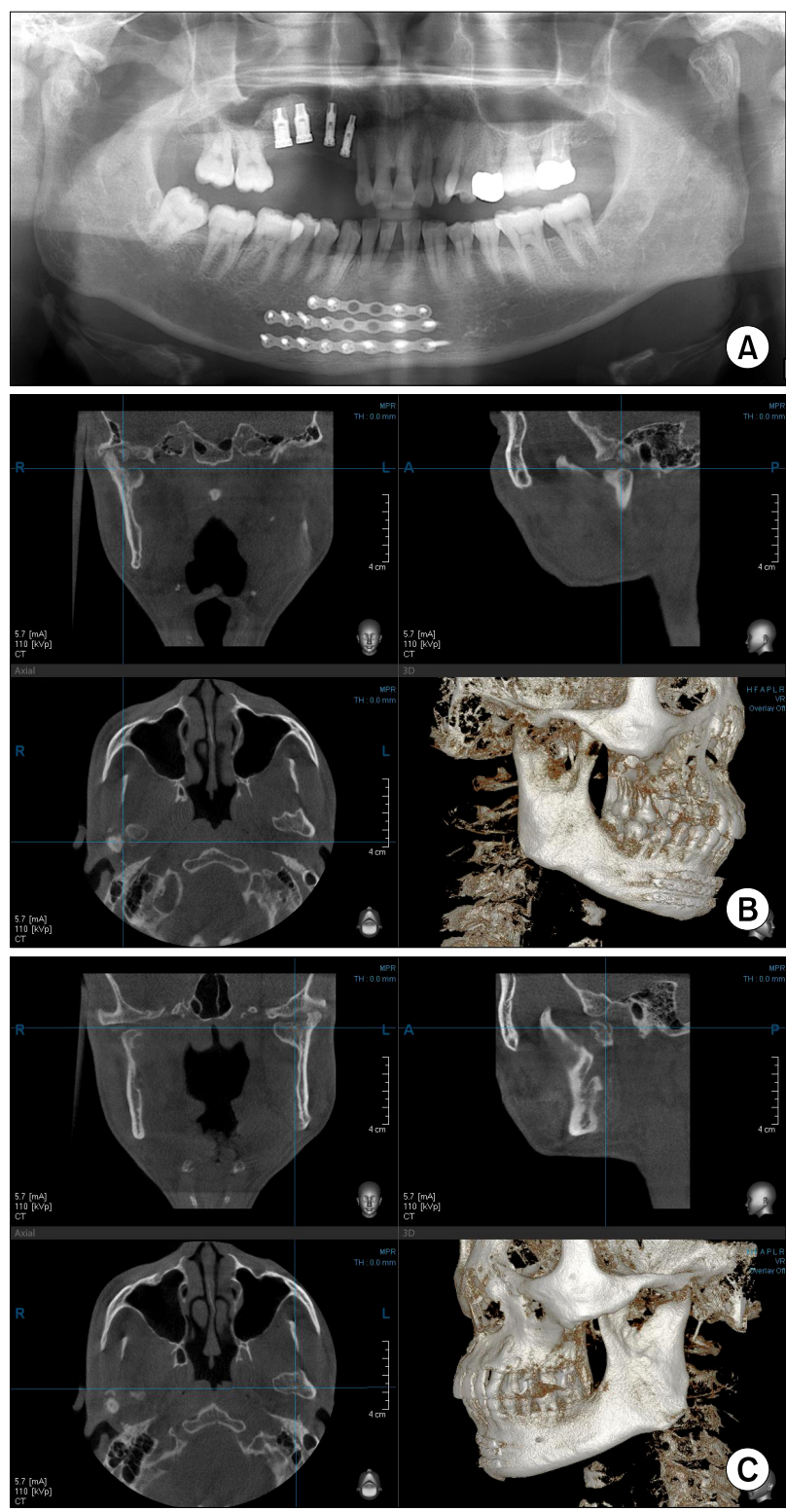

Fig. 2. Preoperative radiograph. (A) Panoramic view. (B) Threedimensional (3D) cone-beam computed tomography (CT) view of the right side. (C) 3D cone-beam CT view of the left side. 
nations revealed pain in the right anterior auricular region when opening the mouth, limited mouth opening with a maximum interincisal opening of $19 \mathrm{~mm}$ (Fig. 1), and crepitation at the right TMJ. On a radiograph and rapid prototyping model, previous condylar fracture fragments and erosive changes were observed around the cleavage at the right mandibular condyle and malunion was observed between fracture fragments at the left mandibular condyle (Fig. 2). Increased uptake around the right condyle region was observed on a bone scan (Fig. 3).

The patient was diagnosed as degenerative osteoarthritis at the right condyle region and resultant limitation of mouth opening due to left and right, especially right, mandibular condylar fracture. To reduce the pain and relieve the limitation of mouth opening, we planned invasive condylectomy and alloplastic total TMJ replacement for re-
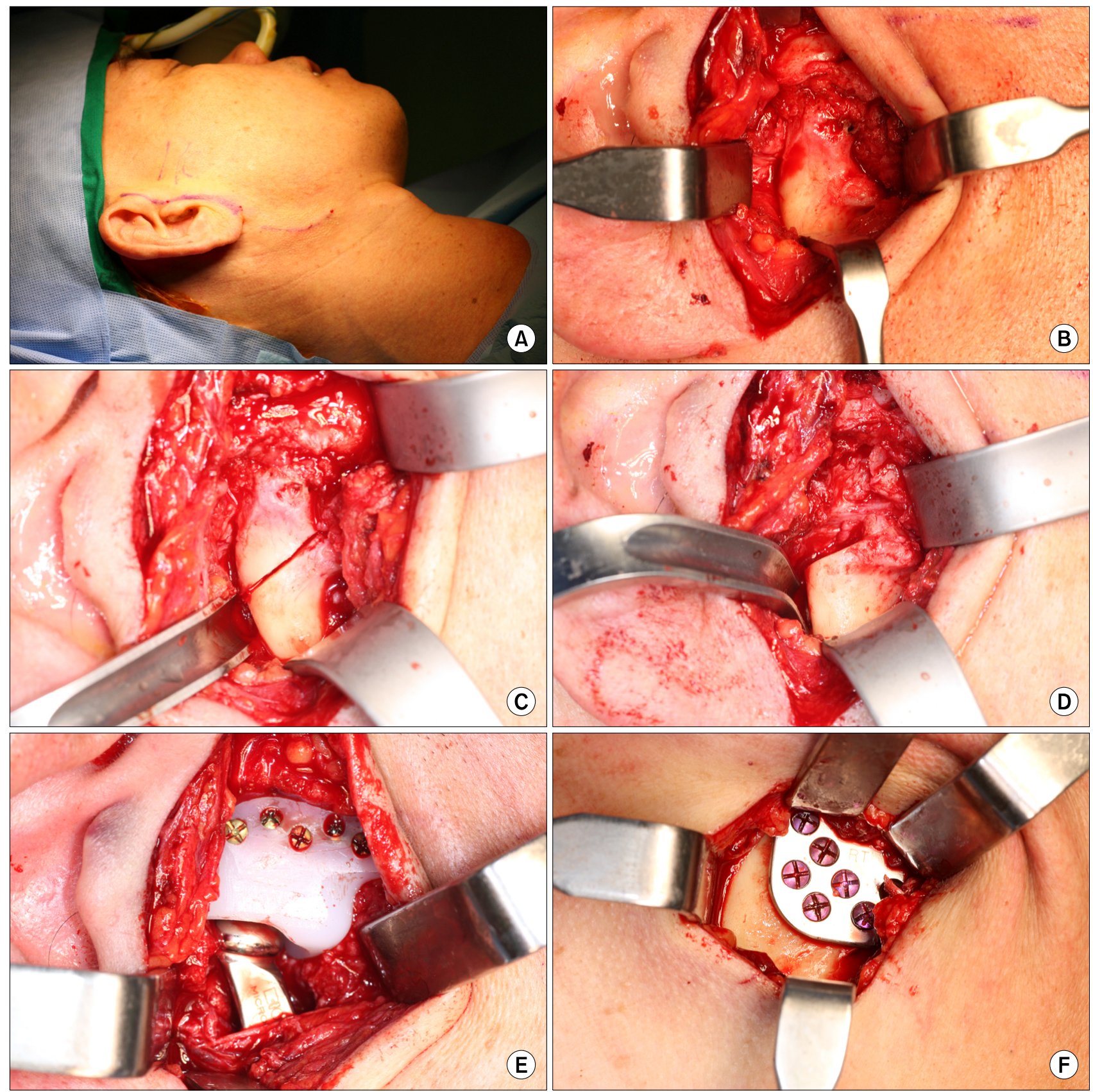

Fig. 4. Operation procedure. (A) Standard endaural and submandibular incision. (B) Exposure of the joint. (C) Begin osteotomy. (D) Removal of condyle and bone fragment. (E) Fitting and placement of the fossa component. (F) Final screw placement of the mandibular component. 
construction of the condyle and fossa, which were affected by degenerative osteoarthritis, using prostheses; a Biomet-Lorenz total TMJ prosthesis (Biomet joint replacement system; Biomet Microfixation, Jacksonville, FL, USA) was used.

Under general anesthesia, the TMJ, condyle, and ramus were exposed through preauricular and submandibular approaches. The right condyle was removed by osteotomy using a reciprocating saw at the sigmoid notch, and the malunited fracture fragments were removed. After removal of the condyle and fracture fragments, fossa for insertion of a fossa component and articular eminence region were flattened using a cylindrical diamond bur, and a fossa implant was placed along the Frankfort Horizontal line and fixed using metal screws at the zygomatic arch. Using the submandibular approach, a medium sized mandibular component was fixed using seven metal screws at the ramus, and the fossa implant was joined by the head of a condylar prosthesis and fixed using six metal screws (Fig. 4).

After the operation, intermaxillary fixation was performed for five days, after which rehabilitation for mouth opening was provided. The patient was discharged one week after the operation, with instructions to continue self-rehabilitation using fingers after discharge. At a visit after three months, the pain in the anterior auricular region and limited mouth opening were resolved, with a maximum interincisal opening of $31 \mathrm{~mm}$, and the joint crepitation had also disappeared (Fig. 5). The amount of mouth opening was maintained over a follow-up period of one year and there was no occurrence of other TMJ complications.

\section{Case 2}

A 46-year-old male patient visited the department complaining of limited mouth opening. Twenty years ago, he underwent gap arthroplasty and coronoidectomy of both TMJ at our hospital for TMJ ankylosis due to rheumatoid arthritis, however, the ankylosis recurred. Clinical examinations revealed complete bilateral adhesions preventing mandibular movements, with a maximum interincisal opening of $7 \mathrm{~mm}$ (Fig. 6). For more accurate diagnosis, clinical examinations as well as panoramic view radiography and cone-beam computed tomography radiography were performed, by which an rapid prototyping (RP) model was generated. On the radiograph, severe adhesion was

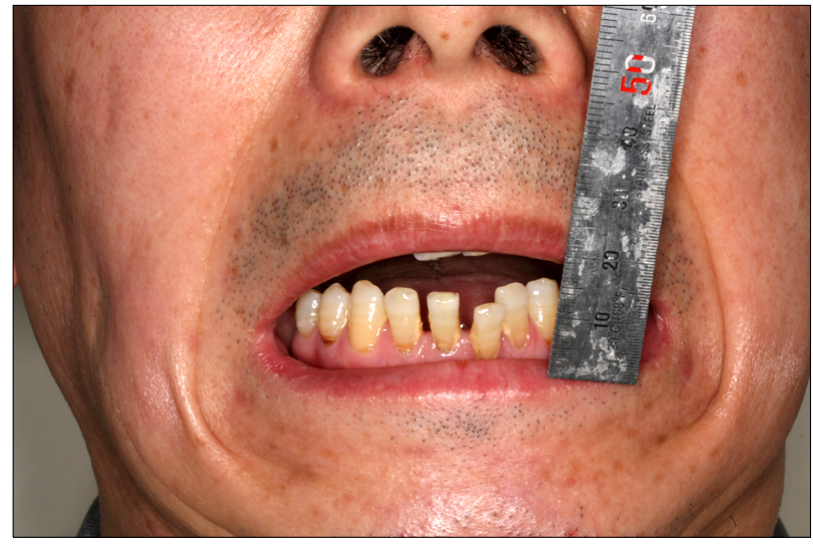

Fig. 6. Preoperative clinical photograph. Maximum interincisal opening was $7 \mathrm{~mm}$.
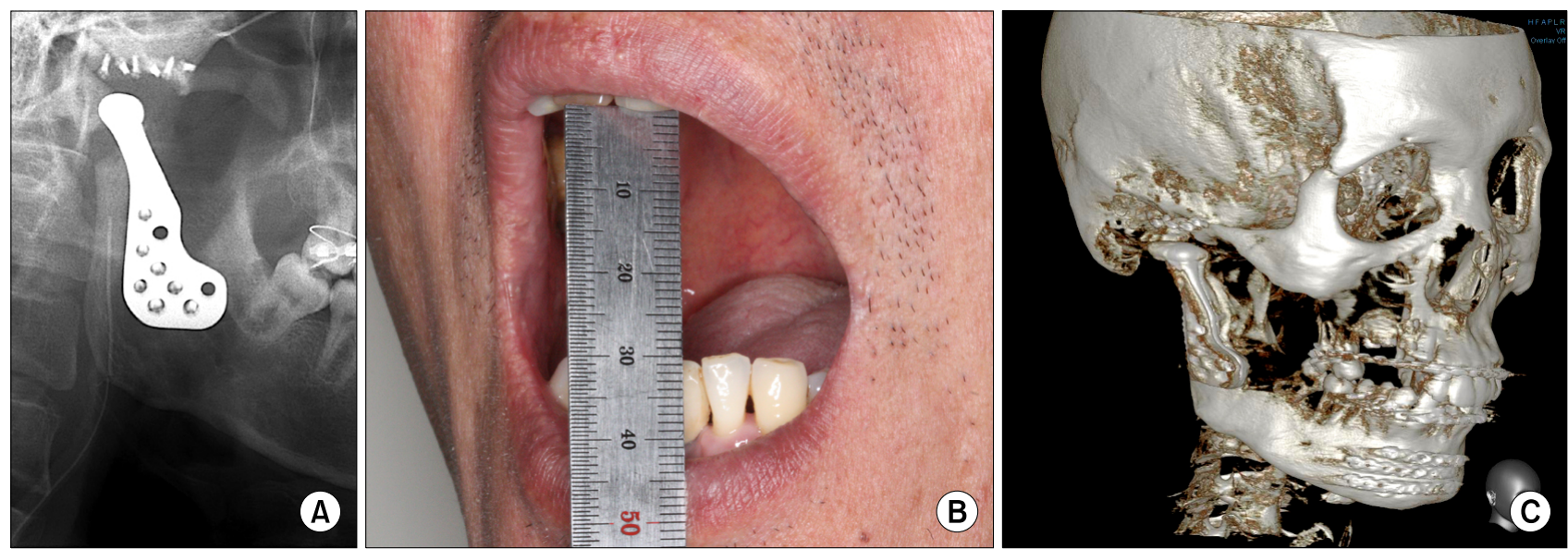

Fig. 5. Postoperative evaluation. (A) Panoramic view. (B) Maximum interincisal opening of $31 \mathrm{~mm}$. (C) Three-dimensional reconstructed cone-beam computed tomography image. 
observed at the bilateral condylar heads and vertical overgrowth was observed at the bilateral coronoid processes (Fig. 7).

The patient was diagnosed as limitation of mouth opening due to ankylosis of the bilateral condyles. A model surgery was performed using the RP model, and an operational plan was then established accordingly (Fig. 8). To alleviate the limited mouth opening, we planned alloplastic total TMJ replacement, accompanied by glenoid reconstruction
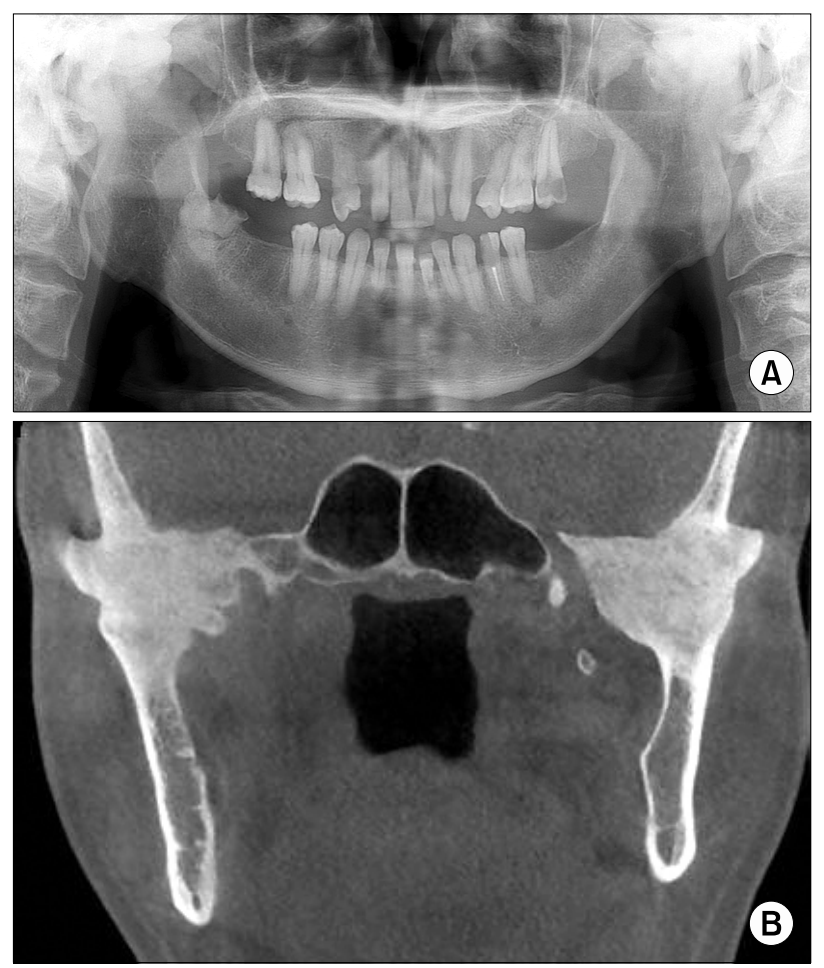

Fig. 7. Preoperative radiograph. (A) Panoramic view. (B) Coronal view of dental cone-beam computed tomography (CT). CT scans showed severe bony ankylosis on both temporomandibular joint. and condylectomy, and bilateral coronoidectomy using an invasive approach.

Under general anesthesia, the TMJ, mandibular condyles, and rami were exposed through a bilateral anterior auricular approach and a submandibular approach. Bilateral mandibular condyles were removed using a reciprocating saw. Maximum opening was improved after removal of the bilateral condylar heads and coronoid processes. Alloplastic joints were fixed in the same manner as described in the first case (Fig. 9).

After the operation, intermaxillary fixation was performed for five days, with mouth opening exercise, and the patient was discharged one week after the operation, at which time the patient showed stable occlusion and a maximum interincisal opening of $23 \mathrm{~mm}$. He has since continued with the mouth opening exercise and ambulatory care (Fig. 10, 11). During the postoperative period of over six months, this patient had no clinically significant symptoms or TMJ problems.

\section{Discussion}

The primary objective of TMJ reconstruction is recovery of anatomical structure and function, and the second objective is to alleviate pain. TMJ reconstruction is generally categorized as either autogenous bone graft or prosthetic material graft. Autogenous bone graft using costal cartilage or fibula is advantageous in biocompatibility and the possibility of growth of the graft but may induce absorption and re-adhesion, overgrowth or discomfort at the recipient site. On the other hand, in a prosthetic material graft, the graft can function within a short period of time without

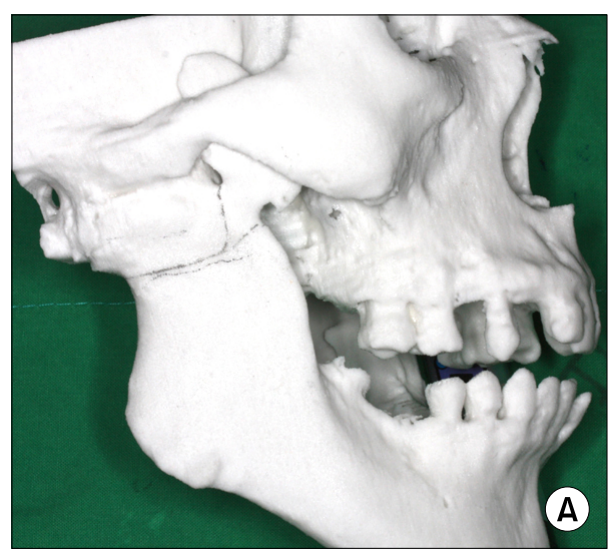

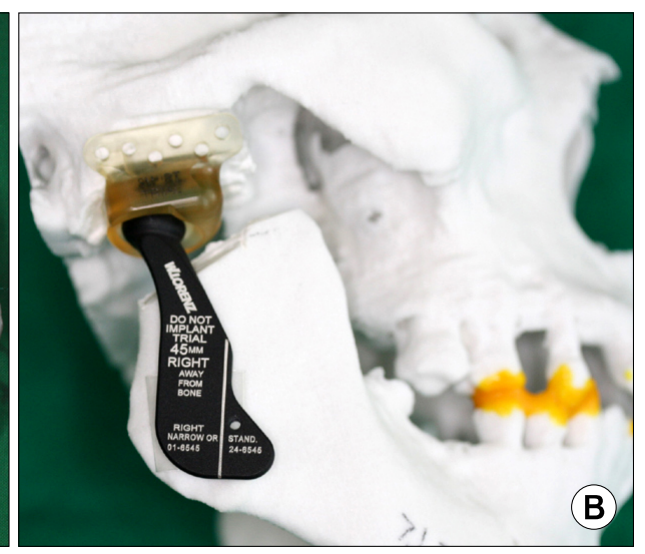

B
Fig. 8. Rapid prototyping model. (A) Planning of the osteotomy lines on the right side of the joint. (B) Mandibular and fossa trial was used to assess the size of the prosthesis. 

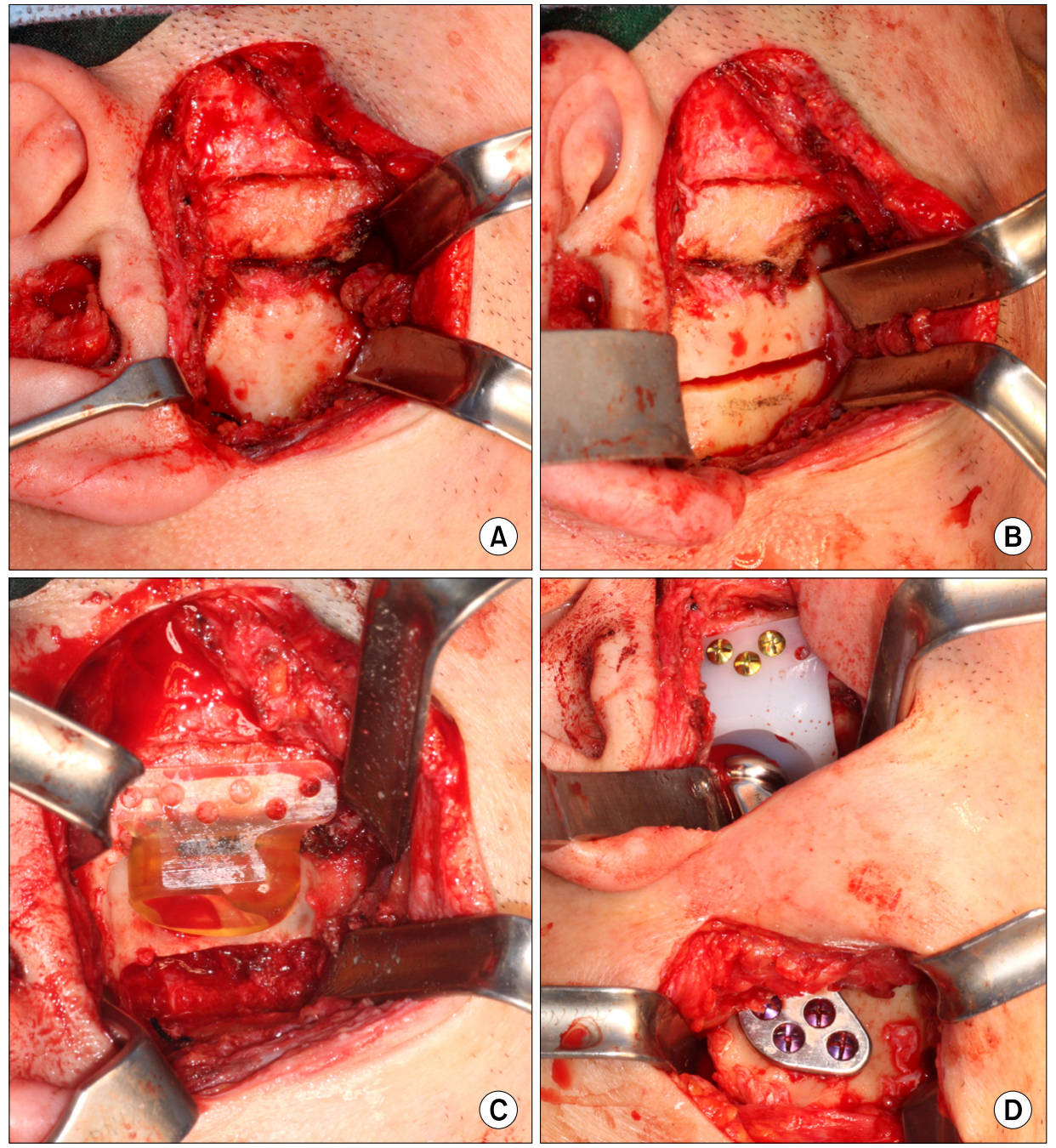

Fig. 9. Operation procedure. (A) Exposure of the joint. (B) Condylectomy cut at the level of the sigmoid notch. (C) Fitting and placement of the fossa trial. (D) Final screw placement of the mandibular component.

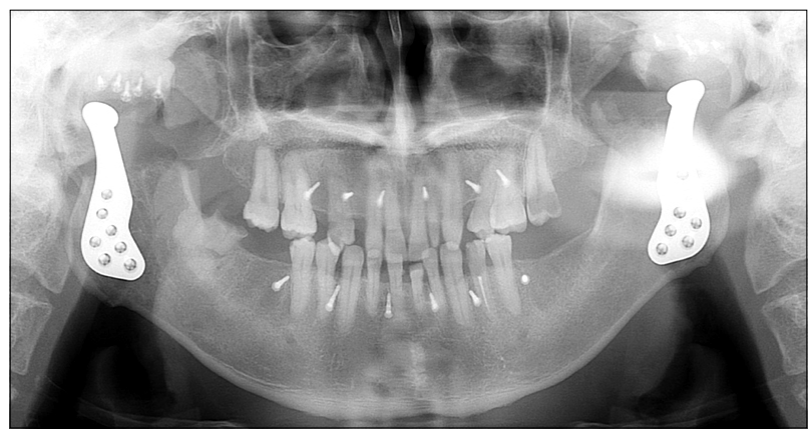

Fig. 10. Postoperative panoramic view.

a postoperative long-term intermaxillary fixation, operation time can be reduced as a recipient site is not required, and the anatomical TMJ can be reconstructed in a form that will prevent ectopic ossification[10].

Since introduction of mandibular resection in the early 19th century, TMJ reconstruction using prosthesis was start-

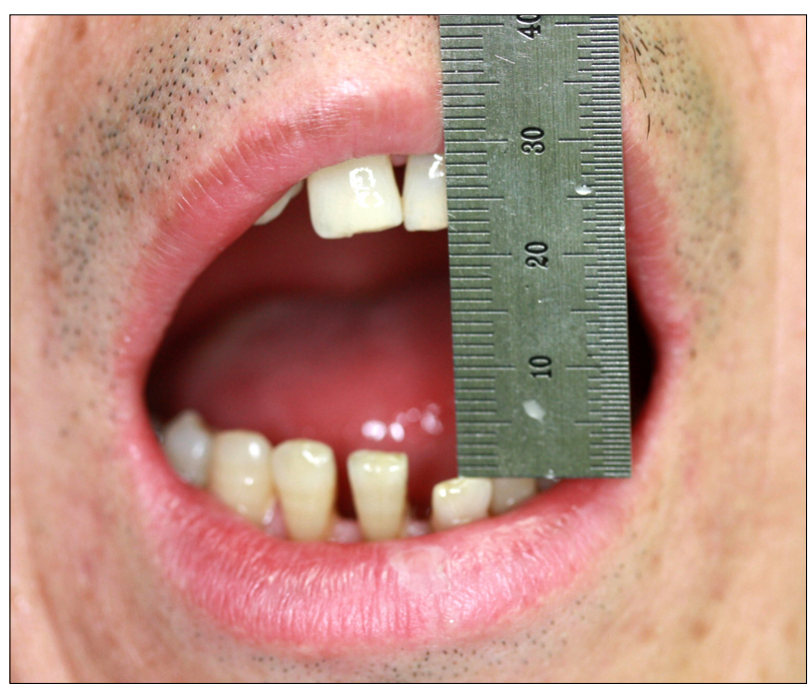

Fig. 11. Postoperative two weeks. Maximum interincisal opening was $23 \mathrm{~mm}$. 
ed by French and German surgeons in an effort to manage complications, such as adhesion and dysfunction, of mandibular resection, including condylar head, and included interpositional alloplastic implant between the condylar head and glenoid fossa and resection dressing and prostheses. However, the focus at that time was mainly on prosthetic reconstruction of the mandibular condylar head, with greater attention to sterilization and biocompatibility, and overall function of TMJ in the oral-facial region was ignored[11]. Glenoid fossa has gained attention in the context of TMJ reconstruction since 1946, when Eggers[12] performed glenoid fossa implant using tantalum foil after surgery for adhesion. In 1968, Robinson[13] introduced partial TMJ reconstruction using glenoid fossa prostheses made of stainless steel. Christensen[14] then produced Chrome-cobalt glenoid fossa, and, in 1971, started using a condylar prosthesis, which consisted of an acrylic head fixed to a vitallium plate.

Since then, with increased knowledge of degenerative arthritis and limitation of partial joint reconstruction, total TMJ replacement has been developed. Kiehn et al.[15] used Chrome-cobalt glenoid fossa and a condylar prosthesis, and Kent et al.[16] detected glenoid erosion causing pain and deformation only when a condylar prosthesis was used for a rheumatoid joint, and performed total TMJ replacement using a Chrome-cobalt condylar head and Fluorinated ethylene propylene Proplast II glenoid fossa. Since then, many studies have investigated various materials and shapes of prostheses and considerable developments have been made, which could maximize both function and biocompatibility. Nowadays, prostheses for TMJ reconstruction are categorized as glenoid fossa prostheses, prosthesis and reconstruction plate for replacement of the condyle, and prosthesis for combined replacement of the fossa and the condyle[17], enabling partial TMJ reconstruction and total TMJ replacement using the most efficient prosthesis depending on various conditions, such as the presence of an articular disc, the degree of degenerative disease development, biomechanic profile of the joint function, and pathophysiology of the joint disease. Cha et al.[18] performed this technique for eight patients, six of whom had idiopathic condylar resorption, and no significant abnormality was observed during follow up. More recently, Kim et al.[19] reported successful results in total joint re- placement reconstruction in a patient with bilateral osseous ankylosis of TMJ and ankylosing spondylitis.

In our case, the prosthesis used for the total TMJ replacement was a Biomet-Lorenz total TMJ prosthesis, which is composed of a glenoid prosthesis made of arcom ultra high molecular weight polyethylene (UHMWPE), condylar prosthesis made of cobalt chromium alloy, and fixing screws made of titanium. Indications for total TMJ replacement using a prosthesis, as in our case, include inflammatory joints such as rheumatoid arthritis, osteoarthritis, and traumatic arthritis; bony adhesion or fibrous adhesion; recurrent adhesion accompanied by excessive ectopic ossification; degenerative joint diseases accompanied by anatomical changes; condylar avascular necrosis; failure of reconstruction by means of allograft implantation such as costal cartilage graft; trauma or benign tumor; loss of condylar head due to treatment of a malignant tumor; and congenital disease.

However, total TMJ replacement is contraindicated in patients with hypersensitivity to prostheses materials or a systemic disease that could easily be affected by infection, such as chronic infection, diabetes mellitus, and myelodysplasia, as well as in patients whose skeletal is still growing.

In case 1 , reconstruction with both glenoid and condylar prostheses was planned for prevention of abnormal adhesion due to the condylar fracture and resultant bone changes; articular disc and retrodiscal tissue damage, and resultant morphological changes on the surfaces of glenoid and condyle, due to severe degenerative arthritis; and additional glenoid absorption in this condition. The right TMJ with degenerative arthritis was approached and condylar resection was performed, followed by removal of pathological tissues and fracture fragments. After five days of postoperative intermaxillary fixation, the patient soon regained function, with a satisfactory maximum interincisal opening and reduced pain. Mandibular deviation when opening the mouth or dislocation of the prosthetic condyle/ glenoid at maximum mouth opening was not observed.

On the other hand, in case 2, total bilateral prosthetic condyle and glenoid reconstruction were performed for the morphological abnormality due to the fracture and adhesion, loss of physiological function, and loss of articular disc. After the bilateral condyle resection, the maximum mouth opening was not satisfactory and the overgrown 
bilateral coronoid process was resected, after which a satisfactory maximum mouth opening was achieved and a satisfactory open mouth pathway was achieved after glenoid and condyle prostheses reconstruction. Although the patient did not have pain or deviation while opening the mouth, limitation in opening the mouth to $23 \mathrm{~mm}$ or more was observed, which might possibly be due to detachment of masticatory muscle, such as temporal muscle, by coronoid process resection and muscular atrophy due to long-term ankylosis. So far, the patient has performed mouth opening exercise using rubber bands.

The current recommendations from the manufacturers suggest positioning the head of the condyle as far posteriorly as possible to allow 'pseudo-translation' of the condyle. If the condyle is too far anteriorly in the closed position, condylar dislocation over the fossa could occur. Preoperative RP model surgery is strongly recommended in order to reduce the possibility of incorrect intraoperative positioning and dislocation.

Selection of an appropriate rehabilitation after the operation had a strong effect on the result of treatment in both cases. Functional rehabilitation started one week after the procedure and aggressive range-of-motion exercise was performed. Each patient opened the mouth for approximately 15 to 30 minutes, with the help of guardians, by gradually increasing the interincisal opening until reaching the maximum interincisal opening. Mouth opening rehabilitation was performed for six weeks after the procedure, and the patients continued the exercise using their fingers after discharge. Then, active exercise therapy was started in order to maintain motility. After releasing intermaxillary fixation, light passive opening and protrusion stretching exercise was performed four times per day for five days. In addition, patients were provided with a soft diet during the first two postoperative weeks.

Although these two cases of total TMJ replacement required long-term follow-up, satisfactory results were obtained in terms of relieving the pain and recovery of function. Few data on prosthetic TMJ reconstruction have been published, however, if more case series are conducted and long-term prognosis, treatment methods and postoperative management were established, alloplastic total TMJ replacement could be widely accepted as an option for treatment of more complex TMJ disorder.

\section{References}

1. Ellis E, Throckmorton GS. Treatment of mandibular condylar process fractures: biological considerations. J Oral Maxillofac Surg 2005;63:115-34.

2. Hlawitschka M, Eckelt U. Assessment of patients treated for intracapsular fractures of the mandibular condyle by closed techniques. J Oral Maxillofac Surg 2002;60:784-91.

3. Hovinga J, Boering G, Stegenga B. Long-term results of nonsurgical management of condylar fractures in children. Int J Oral Maxillofac Surg 1999;28:429-40.

4. Long $\mathrm{X}$, Goss AN. Pathological changes after the surgical creation of a vertical intracapsular condylar fracture. Int J Oral Maxillofac Surg 2007;36:834-7.

5. Sanders B, McKelvy B, Adams D. Aseptic osteomyelitis and necrosis of the mandibular condylar head after intracapsular fracture. Oral Surg Oral Med Oral Pathol 1977;43:665-70.

6. Bear SE, Tankersley RL. Bilateral ankylosis and hyperplasia of the mandibular condyles after mandibular fractures: report of case. J Oral Surg 1971;29:451-5.

7. Park J, Keller EE, Reid KI. Surgical management of advanced degenerative arthritis of temporomandibular joint with metal fossa-eminence hemijoint replacement prosthesis: an 8-year retrospective pilot study. J Oral Maxillofac Surg 2004;62:320-8.

8. Elgazzar RF, Abdelhady AI, Saad KA, et al. Treatment modalities of TMJ ankylosis: experience in Delta Nile, Egypt. Int J Oral Maxillofac Surg 2010;39:333-42.

9. Mercuri LG. Subjective and objective outcomes in patients reconstructed with a custom-fitted alloplastic temporomandibular joint prosthesis. J Oral Maxillofac Surg 1999;57:1427-30.

10. Mercuri LG, Wolford LM, Sanders B, White RD, Giobbie-Hurder A. Long-term follow-up of the CAD/CAM patient fitted total temporomandibular joint reconstruction system. J Oral Maxillofac Surg 2002;60:1440-8.

11. Driemel O, Ach T, Muller-Richter UD, et al. Historical development of alloplastic temporomandibular joint replacement before 1945. Int J Oral Maxillofac Surg 2009;38:301-7.

12. Eggers GW. Arthroplasty of the temporomandibular joint in children with interposition of tantalum foil. J Bone Joint Surg Am 1946;28:603-6.

13. Robinson $\mathrm{M}$. Temporomandibular ankylosis corrected by creating a false silastic sponge fossa. J South Calif Dent Assoc 1968;36:14-6.

14. Christensen RW. The temporomandibular joint prosthesis eleven years later. Oral Implantol 1971;2:125-33.

15. Kiehn CL, DesPrez JD, Converse CF. A new procedure for total temporomandibular joint replacement. Plast Reconstr Surg 1974;53:221-6.

16. Kent JN, Carlton DM, Zide MF. Rheumatoid disease and related arthropathies. II. Surgical rehabilitation of the temporomandibular joint. Oral Surg Oral Med Oral Pathol 1986;61: 423-39.

17. Driemel O, Braun S, Muller-Richter UD, et al. Historical development of alloplastic temporomandibular joint replacement after 1945 and state of the art. Int J Oral Maxillofac Surg 2009;38:909-20.

18. Cha YH, Kim BJ, Lim JH, Park KH, Kim HG, Huh JK. Analysis 
of treatment patterns of temporomandibular disorders. J Korean Assoc Oral Maxillofac Surg 2010;36:520-7.

19. Kim TH, Ryu DM, Lee DW, Jee YJ, Hong SO, Jung JH. A case report of temporomandibular bilateral osseous ankylosis treated by total joint replacement in ankylosing spondylitis. J Korean Assoc Maxillofac Plast Reconstr Surg 2012;34:455-61. 